\title{
AN INFINITE SEQUENCE OF INEQUALITIES INVOLVING SPECIAL VALUES OF THE RIEMANN ZETA FUNCTION
}

\author{
Mircea MERCA
}

Abstract. In this paper, we give an infinite sequence of inequalities involving the Riemann zeta function with even arguments $\zeta(2 n)$ and the Chebyshev-Stirling numbers of the first kind. This result is based on a recent connection between the Riemann zeta function and the complete homogeneous symmetric functions [18]. An interesting asymptotic formula related to the $n$th complete homogeneous symmetric function is conjectured in this context:

$$
h_{n}\left(1,\left(\frac{k}{k+1}\right)^{2},\left(\frac{k}{k+2}\right)^{2}, \ldots\right) \sim\left(\begin{array}{c}
2 k \\
k
\end{array}\right), \quad n \rightarrow \infty .
$$

Mathematics subject classification (2010): 05E05, 11M06, 26D15. functions.

Keywords and phrases: Inequalities, Chebyshev-Stirling number, Riemann zeta function, symmetric

\section{REFERENCES}

[1] M. Abramowitz and I. A. STegun (Eds.), Handbook of Mathematical Functions with Formulas, Graphs, and Mathematical Tables, National Bureau of Standards, Applied Mathematics Series 55, 9th printing, Washington, 1970.

[2] G. E. Andrews And L. L. Littlejohn, A combinatorial interpretation of the Legendre-Stirling numbers, Proceedings AMS, 137, 8 (2009), 2581-2590.

[3] G. E. Andrews, W. Gawronski and L. L. Littlejohn, The Legendre-Stirling numbers, Discrete Math., 311, 14 (2011), 1255-1272.

[4] G. E. Andrews, E. S. EgGe, W. Gawronski and L. L. Littlejohn, The Jacobi-Stirling numbers, J. Combin. Theory, Ser A., 120, 1 (2013), 288-303.

[5] T. M. Apostol, Introduction to Analytic Number Theory, Springer-Verlag, New York-HeidelbergBerlin, 1976

[6] B. C. BERnd, Elementary evaluation of $\zeta(2 n)$, Math. Magazine, 48, 3 (1975), 148-154.

[7] G. Everest, C. RöTtGeR AND T. WARD, The continuing story of zeta, The Math. Intelligencer, 31, 3 (2009), 13-17.

[8] W. N. Everitt, K. H. Kwon, L. L. Littlejohn, R. Wellman And G. J. Yoon, Jacobi-Stirling numbers, Jacobi polynomials, and the left-definite analysis of the classical Jacobi differential expression J. Comput. Appl. Math., 208, 1 (2007), 29-56.

[9] W. Gawronski, L. L. Littlejohn and T. Neuschel, Asymptotics of Stirling and ChebyshevStirling numbers of the second kind, Stud. Appl. Math., 133, 1 (2014), 1-17.

[10] W. Gawronski, L. L. LitTlejohn And T. Neuschel, On the asymptotic normality of the Legendre-Stirling numbers of the second kind, European J. Combin., 49 (2015), 218-231.

[11] Y. GelineaU AND J. ZENG, Combinatorial interpretations of the Jacobi-Stirling numbers Electron. J. Combin. 17 (2010), R70.

[12] I. M. Gessel, Z. Lin And J. Zeng, Jacobi-Stirling polynomials and P-partitions, European J. Combin. 33, 8 (2012), 1987-2000.

[13] K. Ireland And M. Rosen, A Classical Introduction to Modern Number Theory, 2nd ed., Springer, Berlin, 1990 
[14] I. G. Macdonald, Symmetric Functions and Hall Polynomials, 2nd ed., Clarendon Press, Oxford, 1995

[15] M. MercA, A convolution for complete and elementary symmetric functions, Aequat. Math., 86, 3 (2013), 217-229.

[16] M. MerCA, A note on the Jacobi-Stirling numbers, Integral Transforms Spec. Funct., 25, 3 (2014), 196-202.

[17] M. MERCA, A connection between Jacobi-Stirling numbers and Bernoulli polynomials, J. Number Theory., 151 (2015), 223-229.

[18] M. MERCA, Asymptotics of the Chebyshev-Stirling numbers of the first kind, Integral Transforms Spec. Funct., 27, 4 (2016), 259-267.

[19] M. MERCA, The cardinal sine function and the Chebyshev-Stirling numbers, J. Number Theory. 160 (2016), 19-31.

[20] M. MERCA, New convolution for complete and elementary symmetric functions, Integral Transforms Spec. Funct., 27, 12 (2016), 965-973.

[21] P. Mongelli, Total positivity properties of Jacobi-Stirling numbers, Adv. Appl. Math., 48, 2 (2012), 354-364.

[22] P. Mongelli, Combinatorial interpretations of particular evaluations of complete and elementary symmetric functions, Electron. J. Combin., 19, 1 (2012), P60.

[23] M. R. Murty And M. ReECE, A simple derivation of $\zeta(1-K)=-B_{K} / K$, Funct. Approx. Comment. Math., 28 (2000), 141-154.

[24] A. WeIL, Number Theory. An Approach Through History From Hammurapi to Legendre, Birkhäuser, Boston, 1984. 